\title{
F-SPACES UNIVERSAL WITH RESPECT TO LINEAR CODIMENSION
}

\author{
WESLEY E. TERRY
}

\begin{abstract}
Rolewicż raised the question in [5] as to whether there existed a separable $F$-space $X_{0}$ such that any other separable $F$-space $Y$ is the image of $X_{0}$ under a continuous linear operator. This can be equivalently phrased as the question [5, Problem II.4.3, p. 47]: Does there exist a separable $F$-space universal for all separable $F$-spaces with respect to linear codimension? Theorem 1 proves the existence of such a separable $F$-space. Theorem 2 generalizes this idea to larger cardinals.
\end{abstract}

1. Introduction. A metric linear space $X$ is called an $F$-space if $X$ has a complete metric $\rho$ with the property that $\rho(x+z, y+z)=\rho(x, y)$ for all $x$, $y, z \in X$. Such a metric, $\rho$, is said to be invariant. By a theorem of Kakutani [5, Theorem I.1.1, p. 12], every metric linear space has an invariant metric. By a theorem of Klee [5, I.4.3, p. 24] if $X$ is a topologically complete metric linear space then an invariant metric for the topology on $X$ is a complete metric on $X$.

Let $\Lambda$ be a set of cardinality $\aleph$. The space $l_{1}(\aleph)$ is defined to be the set of all real functions $r=\left\{r_{\lambda}\right\}$ defined on the set $\Lambda$ with at most a countable number of nonzero elements and with $\Sigma_{\lambda}\left|r_{\lambda}\right|<\infty$. The norm on $l_{1}(\boldsymbol{*})$ is $\|r\|=\Sigma_{\lambda}\left|r_{\lambda}\right|$.

Schauder proved the following theorem:

Thęrem (Schauder, see Banach-Mazur [1, p. 111] and Klee [4, Proposition 2.1]). Every Banach space $B$, with the weight of $B$ less than or equal to $N$, is a linear image of $l_{1}(\boldsymbol{*})$.

Theorems 1 and 2 are motivated by this result. Theorem 3 proves that the universal spaces of Theorems 1 and 2 are homeomorphic to $l_{1}(\aleph)$ for appropriate cardinal $\kappa$.

\section{Proofs of Theorems.}

THEOREM 1. There is a separable F-space universal with respect to linear codimension for all separable $F$-spaces.

Proof. Let $H(I)$ be the space of all homeomorphisms of the unit interval $I=[0,1]$ onto itself that are the identity on the endpoints and satisfy the following property:

Received by the editors May 17, 1976.

AMS (MOS) subject classifications (1970). Primary 46A15, 54B99; Secondary 57A17. 
If $a, b, c \in[0,1]$ with $a \leqslant b+c$, then $F(a) \leqslant F(b)+F(c)$ for $F \in$ $H(I)$. The metric on $H(I)$ is the supremum metric.

Pick a countable dense subset of $H(I)$. Call it $\left\{g_{i}\right\}_{i=1}^{\infty}$. Pick the rationals in $(0,1]$ and enumerate them as $\left\{p_{i}\right\}_{i=1}^{\infty}$. Let $\left\{f_{i}\right\}_{i=1}^{\infty}$ be an enumeration of the countable number of elements resulting from replacing each $g_{i}$ in $\left\{g_{i}\right\}_{i=1}^{\infty}$ by the countable number of elements $\left\{p_{j} g_{i}\right\}_{j=1}^{\infty}$.

Define $h$ to be the homeomorphism

$$
h:[0, \infty) \rightarrow[0,1), \quad h(r)=r /(1+r) .
$$

Then each $f_{i}$ induces an invariant, strictly monotone metric on $\mathbf{R}=(-\infty, \infty)$ defined by

$$
d_{i}(r, s)=f_{i}(h(|r-s|)) .
$$

$d_{i}$ is a metric since $f_{i}=p_{j} g_{k}$ for some $g_{k} \in H(I)$ and $h(a) \leqslant h(b)+h(c)$ if $a \leqslant b+c, a, b, c \in[0, \infty) . d_{i}(t, 0)<d_{i}(s, 0)$ if $0 \leqslant t<s$, since both $h$ and $f_{i}$ are strictly monotone. Finally,

$$
\begin{aligned}
d_{i}(t+s, r+s)= & f_{i}(h(|t+s-(r+s)|)) \\
& =f_{i}(h(|t-r|))=d_{i}(t, r)
\end{aligned}
$$

for all $t, r, s \in \mathbf{R}$. Thus $d_{i}$ is an invariant metric.

Now, let $\prod_{j=1}^{\infty} \mathbf{R}_{j}$ be the countable cartesian product of copies of $\mathbf{R}$. Define

$$
\sum_{l_{1}}\left(\mathbf{R}, d_{j}\right)=\left\{\left\{x_{j}\right\} \in \prod_{j=1}^{\infty} \mathbf{R}_{j} \mid\left\{d_{j}\left(x_{j}, 0\right)\right\}_{j=1}^{\infty} \in l_{1}\right\} .
$$

Here $l_{1}=l_{1}\left(\kappa_{0}\right)$. Then $\sum_{l_{1}}\left(\mathbf{R}, d_{j}\right)$ is an $F$-space under coordinatewise addition and scalar multiplication and under the invariant metric $\sum_{j=1}^{\infty} d_{j}\left(x_{j}, y_{j}\right)=$ $\rho\left(\left\{x_{j}\right\},\left\{y_{j}\right\}\right)$. Given this invariant metric $\rho$,

$$
\left|\left\{x_{j}\right\}\right|_{l_{1}}=\sum_{j=1}^{\infty} d_{j}\left(x_{j}, 0\right)
$$

is called the associated $F$-norm. [Given an invariant metric $\rho$ on an $F$-space, the associated $F$-norm is defined to be ||$=\rho(\cdot, 0)$.]

We claim that $\left(\Sigma_{l_{1}}\left(\mathbf{R}, d_{j}\right),||_{l_{1}}\right)$ is the required universal $F$-space. $\Sigma_{l_{1}}\left(\mathbf{R}, d_{j}\right)$ is clearly separable. Given a separable $F$-space $(M,||)$, we may assume that the $F$-norm is not only invariant but also strictly monotone on rays from the origin and bounded by one. (See Eidelheit and Mazur [3].) Pick a countable dense subset of $M$ from $M \backslash\{0\}$, and call this collection $\left\{x_{i}\right\}_{i=1}^{\infty}$.

Look at the lines $L_{i}=\left\{r x_{i} \mid r \in \mathbf{R}\right\}, i=1,2, \ldots$ Then, for each $i,\left(L_{i},||_{i}\right)$ is an $F$-space where ||$_{i}$ is || restricted to $L_{i}$. Let

$$
s_{i}=\sup _{s \in(0, \infty)}\left|s x_{i}\right|_{i} \leqslant 1 \text {. }
$$

Then ||$_{i}$ takes on values in $\left[0, s_{i}\right)$ since $|-x|=|x|$ by definition of an $F$-norm and || is strictly monotone so that ||$_{i}$ cannot take on the supremum.

Define $F_{i}:\left[0, s_{i}\right] \rightarrow\left[0, s_{i}\right]$ by 


$$
F_{i}(r)= \begin{cases}\left|\left(r /\left(s_{i}-r\right)\right) x_{i}\right|_{i}, & r \neq s_{i} \\ s_{i}, & r=s_{i}\end{cases}
$$

Then $F_{i}$ is a homeomorphism of $\left[0, s_{i}\right]$ onto itself since $\|$ is strictly monotone. Thus $F\left(s_{i} \cdot\right) / s_{i} \in H(I)$ since ||$_{i}$ satisfies the triangle inequality and is strictly monotone. Also, $F_{i}\left(s_{i} \cdot\right)$ is a homeomorphism of $[0,1]$ onto $\left[0, s_{i}\right]$.

Now, for $F_{1}\left(s_{1} \cdot\right)$ pick an element in $\left\{f_{j}\right\}_{j=1}^{\infty}$ such that

$$
\sup _{r \in[0,1]}\left|F_{1}\left(s_{1} r\right)-f_{\sigma(1)}(r)\right|<\frac{1}{2} \text {. }
$$

Then inductively select an element in $\left\{f_{j}\right\}_{j=1}^{\infty} \backslash\left\{f_{\sigma(1)}, \ldots, f_{\sigma(i-1)}\right\}$ for $F_{i}\left(s_{i} \cdot\right)$ such that $\sup _{r \in[0,1]}\left|F_{i}\left(s_{i} r\right)-f_{\sigma(i)}(r)\right|<1 / 2^{i}$. This can be done since rational scalar multiples of the $\left\{g_{i}\right\}_{i=1}^{\infty}$ have been included in $\left\{f_{i}\right\}_{i=1}^{\infty}$.

Define a linear operator $A$ from $\Sigma_{l_{1}}\left(\mathbf{R}, d_{j}\right)$ to $M$ by defining it on the Schauder basis $\left\{\delta_{j}\right\}_{j=1}^{\infty}$ where

$$
\begin{gathered}
\delta_{j}=\left\{\begin{array}{ll}
0, & i \neq j, \\
1, & i=j,
\end{array} \quad \delta_{j} \in \sum_{l_{1}}\left(\mathbf{R}, d_{j}\right),\right. \\
A: \sum_{l_{1}}\left(\mathbf{R}, d_{j}\right) \rightarrow M, \quad A\left(\delta_{j}\right)= \begin{cases}0, & j \neq \sigma(i) \text { for any } i, \\
x_{j}, & j=\sigma(i) \text { for some } i .\end{cases}
\end{gathered}
$$

We claim that $A$ is a continuous linear surjection.

$A$ is into since $F_{i}\left(s_{i} r\right)<1 / 2^{i}+f_{\sigma(i)}(r)$ and, therefore, $\left|r x_{i}\right|_{i}<1 / 2^{i}+$ $d_{\sigma(i)}(r, 0)$ for each $i$ and any $r \in[0,1]$. Hence

$$
\begin{aligned}
\sum_{i=1}^{\infty}\left|r_{\sigma(i)} x_{i}\right|_{i} & <\sum_{i=1}^{\infty}\left[\frac{1}{2^{i}}+d_{\sigma(i)}\left(r_{\sigma(i)}, 0\right)\right] \\
& =1+\sum_{i=1}^{\infty} d_{\sigma(i)}\left(r_{\sigma(i)}, 0\right) \\
& <\infty \quad \text { for }\left\{r_{j}\right\} \in \sum_{l_{1}}\left(\mathbf{R}, d_{j}\right)
\end{aligned}
$$

Note that the induced $F$-norm on $\mathbf{R}$ induced by $F_{i}\left(s_{i} \cdot\right)$ is $d(r, t)=$ $F_{i}\left(s_{i} h(|r-t|)\right)$. Hence

$$
d(r, t)=F_{i}\left(s_{i} h(|r-t|)\right)=\left|(r-t) x_{i}\right|_{i}
$$

To see that $A$ is continuous, let $\left\{r_{j}\right\},\left\{r_{j}^{n}\right\} \in \Sigma_{l_{1}}\left(\mathbf{R}, d_{j}\right), n=1,2, \ldots$, and suppose $\left\{r_{j}^{n}\right\} \rightarrow\left\{r_{j}\right\}$. Then look at the indices corresponding to $\sigma(i), i=1$, $2, \ldots$ All other coordinates go to zero under $A$. Then

$$
\sum_{i=1}^{\infty} d_{\sigma(i)}\left(r_{\sigma(i)}^{n}, r_{\sigma(i)}\right) \leqslant \sum_{j=1}^{\infty} d_{j}\left(r_{j}^{n}, r_{j}\right) .
$$

Now, given $\varepsilon>0$, choose $N_{0}$ so that $\sum_{N_{0}+1}^{\infty} 1 / 2^{n}<\varepsilon / 4$. Next, find each index of the form $\sigma(i)$ such that $\sigma(i) \leqslant N_{0}$. Call these indices $k_{1}=\sigma\left(i_{1}\right), \ldots, k_{t}=$ $\sigma\left(i_{t}\right)$. Then, given $\varepsilon / 2 N_{0}$, pick a $\delta$ where $0<\delta<\varepsilon / 4$ such that $F_{i_{j}}\left(s_{i_{j}} r\right)<$ $\varepsilon / 2 N_{0}$ whenever $d_{k_{i}}(r, 0)<\delta, j=1,2, \ldots, t$. Now, given $\delta>0$, pick $N_{1} \geqslant N_{0}$ such that $\sum_{j=1}^{\infty}\left(r_{j}^{n}, r_{j}\right)<\delta$ whenever $n \geqslant N_{1}$. Then for $n \geqslant N_{1}$, 


$$
\begin{aligned}
\left|A\left(\left\{r_{j}^{n}\right\}\right)-A\left(\left\{r_{j}\right\}\right)\right|=\left|\sum_{i=1}^{\infty} r_{\sigma(i)}^{n} x_{i}-\sum_{i=1}^{\infty} r_{\sigma(i)} x_{i}\right| \\
\leqslant \sum_{i=1}^{\infty}\left|\left(r_{\sigma(i)}^{n}-r_{\sigma(i)}\right) x_{i}\right| \\
\leqslant \sum_{j=1}^{t}\left|\left(r_{k_{j}}^{n}-r_{k_{j}}\right) x_{i_{j}}\right|+\sum_{\sigma(i)>N_{0}+1}\left|\left(r_{\sigma(i)}^{n}-r_{\sigma(i)}\right) x_{i}\right| \\
<N_{0}\left(\frac{\varepsilon}{2 N_{0}}\right)+\sum_{\sigma(i)>N_{0}+1}\left[d_{\sigma(i)}\left(r_{\sigma(i)}^{n}, r_{\sigma(i)}\right)+\frac{1}{2^{i}}\right] \\
<\varepsilon / 2+\varepsilon / 4+\varepsilon / 4=\varepsilon .
\end{aligned}
$$

To see that $A$ is surjective, let $y \in M$. Again, look at $\left\{x_{i}\right\}_{i=1}^{\infty}$, the countable dense set in $M$. Pick $r_{\beta(1)} x_{\beta(1)}$ such that $\left|y-r_{\beta(1)} x_{\beta(1)}\right|<\frac{1}{2}$. Next, since $\left\{x_{i}\right\}_{i=1}^{\infty} \backslash\left\{x_{\beta(1)}\right\}$ is still dense in $M$, pick $r_{\beta(2)} x_{\beta(2)}$ so that $\mid\left(y-r_{\beta(1)} x_{\beta(1)}\right)-$ $r_{\beta(2)} x_{\beta(2)} \mid<\frac{1}{4}$. Inductively, pick $r_{\beta(n)} x_{\beta(n)}$ where $x_{\beta(n)}$ is chosen from

$$
\left\{x_{i}\right\}_{i=1}^{\infty} \backslash\left\{x_{\beta(1)}, \ldots, x_{\beta(n-1)}\right\}
$$

so that

$$
\left|\left(y-r_{\beta(1)} x_{\beta(1)}-\cdots-r_{\beta(n-1)} x_{\beta(n-1)}\right)-r_{\beta(n)} x_{\beta(n)}\right|<1 / 2^{n} .
$$

Then

$$
\begin{aligned}
\left|r_{\beta(1)} x_{\beta(1)}\right| & \leqslant\left|y-r_{\beta(1)} x_{\beta(1)}\right|+|y|<\frac{1}{2}+|y|<\frac{1}{2}+1, \\
\left|r_{\beta(2)} x_{\beta(2)}\right| & \leqslant\left|\left(y-r_{\beta(1)} x_{\beta(1)}\right)-r_{\beta(2)} x_{\beta(2)}\right|+\left|y-r_{\beta(1)} x_{\beta(1)}\right| \\
< & \frac{1}{4}+\frac{1}{2} \\
& \vdots \\
& \\
\left|r_{\beta(n)} x_{\beta(n)}\right| & \leqslant\left|\left(y-r_{\beta(1)} x_{\beta(1)}-\cdots-r_{\beta(n-1)} x_{\beta(n-1)}\right)-r_{\beta(n)} x_{\beta(n)}\right| \\
& +\left|\left(y-r_{\beta(1)} x_{\beta(1)}-\cdots-r_{\beta(n-2)} x_{\beta(n-2)}\right)-r_{\beta(n-1)} x_{\beta(n-1)}\right| \\
& <1 / 2^{n}+1 / 2^{n-1}
\end{aligned}
$$

Now, $\sum_{k=1}^{\infty} r_{\beta(k)} x_{\beta(k)} \in M$ since

$$
\left|\sum_{k=1}^{\infty} r_{\beta(k)} x_{\beta(k)}\right| \leqslant \sum_{k=1}^{\infty}\left|r_{\beta(k)} x_{\beta(k)}\right|<|y|+2 \sum_{k=1}^{\infty} \frac{1}{2^{k}}=|y|+2 .
$$

Select $\left\{r_{j}\right\} \in \Sigma_{l_{1}}\left(\mathbf{R}, d_{j}\right)$ by 


$$
r_{j}= \begin{cases}r_{\beta(k)} & \text { if } j=\sigma(\beta(k)) \text { for some } k, \\ 0 & \text { if } j \neq \sigma(\beta(k)) \text { for any } k .\end{cases}
$$

To see that $\left\{r_{j}\right\} \in \Sigma_{l_{1}}\left(\mathbf{R}, d_{j}\right)$ note that

$$
\sup _{r \in[0,1]}\left|F_{\beta(k)}\left(S_{\beta(k)} r\right)-f_{\sigma(\beta(k))}(r)\right|<\left(\frac{1}{2}\right)^{\beta(k)} .
$$

Thus, since $d_{\sigma(\beta(k))}\left(r_{\beta(k)}, 0\right)=f_{\sigma(\beta(k))}\left(h\left(r_{\beta(k)}\right)\right)$,

$$
\begin{aligned}
d_{\sigma(\beta(k))}\left(r_{\beta(k)}, 0\right) & \leqslant(1 / 2)^{\beta(k)}+F_{\beta(k)}\left(s_{\beta(k)} h\left(r_{\beta(k)}\right)\right) \\
& =(1 / 2)^{\beta(k)}+\left|r_{\beta(k)} x_{\beta(k)}\right|<(1 / 2)^{\beta(k)}+1 / 2^{k}+1 / 2^{k-1} .
\end{aligned}
$$

Therefore,

$$
\begin{aligned}
\sum_{j=1}^{\infty} d_{j}\left(r_{j}, 0\right) & =\sum_{k=1}^{\infty} d_{\sigma(\beta(k))}\left(r_{\beta(k)}, 0\right) \\
& \leqslant \sum_{k=1}^{\infty}\left[\left(\frac{1}{2}\right)^{\beta(k)}+\frac{1}{2^{k}}+\frac{1}{2^{k-1}}\right] \leqslant 1+3<\infty .
\end{aligned}
$$

Clearly, $A\left(\left\{r_{j}\right\}\right)=\sum_{k=1}^{\infty} r_{\beta(k)} x_{\beta(k)}=y$. Hence $A$ is surjective.

Thus $A$ has all the properties required, and $\Sigma_{l_{1}}\left(\mathbf{R}, d_{j}\right)$ satisfies the conditions of the theorem.

THEOREM 2. Given an infinite cardinal number $\mathrm{N} \geqslant 2^{\aleph_{0}}$, there is an F-space of weight $\times$ universal with respect to linear codimension for all $F$-spaces of weight less than or equal to $\mathrm{k}$.

Proof. Let $H(I)$ be as in Theorem 1 . Then the cardinality of $H(I)$ is $2^{\mathrm{\kappa}_{0}}$. Let $\left\{g_{t}\right\}_{t \in \mathbf{R}}$ be an enumeration of the elements of $H(I)$. Also, the cardinality of $(0,1]$ is $2^{\aleph_{0}}$ as well. Let $\left\{f_{t}\right\}_{t \in \mathbf{R}}$ be a new enumeration containing $2^{\aleph_{0}}$ elements obtained by replacing each $g_{t}$ by the $2^{\aleph_{0}}$ elements $\left\{r g_{t}\right\}_{r \in(0,1]}$.

Then each $f_{t}$ induces an invariant, strictly monotone metric on $\mathbf{R}$ defined by $d_{t}(r, s)=f_{t}(h(|r-s|))$ just as in Theorem 1 . Note that $d_{t}$ is bounded by one. This gives all possible metrics on $\mathbf{R}$ with these properties.

Now, let $\Pi_{\lambda \in \Lambda} \mathbf{R}_{\lambda}$ be the cartesian product of $*$ copies of $\mathbf{R}$. Here, the cardinality of $\Lambda$ is $\kappa$. Define

$$
\sum_{l_{1}(\boldsymbol{*})}\left(\mathbf{R}, d_{t}\right)=\left\{\left\{x_{t \lambda}\right\} \in \prod_{t \in \mathbf{R}}\left(\prod_{\lambda \in \Lambda} \mathbf{R}_{\lambda}\right) \mid\left\{d_{t}\left(x_{t \lambda}, 0\right)\right\} \in l_{1}(\boldsymbol{\kappa})\right\}
$$

where $(t, \lambda)$ is suitably re-indexed to give a correspondence with the $N$ coordinates of $l_{1}(\kappa)$. Then $\Sigma_{l_{1}(\kappa)}\left(\mathbf{R}, d_{t}\right)$ is an $F$-space under coordinates addition and scalar multiplication, and under the $F$-norm $\left|\left\{x_{t \lambda}\right\}\right|_{l_{1}}=$ $\sum_{t \lambda} d_{t}\left(x_{t \lambda}, 0\right)$. This can be shown to be the required universal space by going through the same process as in Theorem 1. However, when you get to the part where you pick $f_{\sigma(t)}$ for $F_{t}\left(s_{t} \cdot\right)$, you can pick $f_{\sigma(t)}$ equal to $F_{t}\left(s_{t} \cdot\right)$ instead of just near $F_{t}\left(s_{t} \cdot\right)$. Therefore, you have the restricted metric || , from || as the 
chosen induced metric. This, in fact, makes it easier to show that the linear map $A$ is into and continuous. Since each metric is repeated $\times$ times, you can still insure that you choose different directions from the origin in constructing $A$ and in showing that $A$ is onto. Thus, the theorem follows.

THEOREM 3. $\Sigma_{l_{1}(\kappa)}\left(\mathbf{R}, d_{t}\right)$ is homeomorphic to $l_{1}(\aleph)$.

Proof. Define

$$
H: \sum_{l_{1}(*)}\left(\mathbf{R}, d_{t}\right) \rightarrow \sum_{l_{1}(*)}\left(\mathbf{R}, \frac{s_{t}\|\|}{1+\|\|}\right) \text { by } h\left(\left\{r_{\alpha}\right\}\right)=\left\{q_{\alpha} r_{\alpha}\right\}
$$

where each $q_{\alpha}$ is chosen so that when $r_{\alpha} \neq 0, q_{\alpha}$ is the unique positive real number such that

$$
d_{t}\left(q_{\alpha} r_{\alpha}, 0\right)=s_{t}\left\|q_{\alpha} r_{\alpha}\right\| /\left(1+\left\|q_{\alpha} r_{\alpha}\right\|\right)
$$

$H$ sends a zero coordinate to the corresponding zero coordinate. Here, $s_{t}=\sup _{r \in(0, \infty)} d_{t}(r, 0)$ and $\|\cdot\|$ is the absolute value on $\mathbf{R}$. A unique such $q_{\alpha}$ exists for each $\alpha$ when $r_{\alpha} \neq 0$ since $d_{t}$ and $s_{t}\|\cdot\| /(1+\|\cdot\|)$ are both strictly monotone on rays from the origin. $H$ is thus a homeomorphism.

Now, for the separable case, let $\left\{p_{i}\right\}_{i=1}^{\infty}$ be the enumeration of the rationals in $(0,1]$ used in Theorem 1 . Then, for each $i, M_{i}=\sum_{l_{1}}\left(\mathbf{R}, p_{i}\|\| /(1+\|\|)\right)$ is homeomorphic to $l_{1}$ by the identity map. The argument is similar to that of Lemma 6 of [6]. But then, by re-indexing the coordinates of

$$
\sum_{l_{1}}\left(\mathbf{R}, s_{t}\|\| /(1+\|\|)\right)
$$

we have

$$
\sum_{l_{1}}\left(\mathbf{R}, \frac{s_{t}\|\|}{1+\|\|}\right)=\sum_{l_{1}}\left[M_{i}, \sum_{k=1}^{\infty}\left[\frac{p_{i}\|\|}{1+\|\|}\right]_{k}\right] .
$$

Finally, $\Sigma_{l_{1}}\left(M_{i}, \sum_{k=1}^{\infty}\left[p_{i}\|\| /(1+\|\|)\right]_{k}\right)$ is homeomorphic to $l_{1}$ by Theorem 3 of [6] and the fact that $l_{1}$ is homeomorphic to $\prod_{i=1}^{\infty}\left(l_{1}\right)_{i}$. (Bessaga proved in [2] that $l_{1}(\aleph)$ is homeomorphic to $\prod_{i=1}^{\infty}\left(l_{1}(\aleph)\right)_{i}$.)

For the nonseparable case, let $\delta_{i}=\left(1 / 2^{i}, 1 / 2^{i-1}\right]$ for $i=1,2, \ldots$ For each $i$ define

$$
M_{i}=\left\{\left\{r_{s \lambda}\right\} \in \prod_{s \in \delta_{i}}\left(\prod_{\lambda \in \Lambda} \mathbf{R}_{\lambda}\right) \mid \sum_{s} \sum_{s \in \delta_{i}} \sum_{\lambda \in \Lambda} \frac{s\left\|r_{s \lambda}\right\|}{1+\left\|r_{s \lambda}\right\|}<\infty\right\}
$$

with the obvious metric. The argument now proceeds as in the separable case. Each $M_{i}$ is homeomorphic to $l_{1}(\kappa)$ and this implies $\Sigma_{l_{1}(x)}\left(\mathbf{R}, s_{t}\|\| /(1+\|\|)\right)$ is homeomorphic to $l_{1}(\aleph)$.

\section{REFERENCES}

1. S. Banach and S. Mazur, Zur Theorie der linearen Dimension, Studia Math. 4 (1933), 100-112. 
2. Cz. Bessaga, On topological classification of complete linear metric spaces, Fund. Math. 56 (1964/65), 251-288. MR 31 \#2580.

3. M. Eidelheit and S. Mazur, Eine Bemerkung über die Räume vom typus $(F)$, Studia Math. 7 (1938), 159-161.

4. V. L. Klee, Mappings into normed linear spaces, Fund. Math. 49 (1960/61), 25-34. MR 23 \# A3985.

5. S. Rolewicz, Metric linear spaces, Monografie Mat., Tom 56, PWN, Warsaw, 1972.

6. W. E. Terry, Conditions for a TVS to be homeomorphic with its countable product, Trans. Amer. Math. Soc. 190 (1974), 233-242. MR 49 \#3489.

Department of Mathematical Sciences, New Mexico State University, Las Cruces, NeW MeXICO 88003 